\title{
Stronger Association of Albuminuria with the Risk of Vascular Complications than Estimated Glomerular Filtration Rate in Type 2 Diabetes
}

\author{
Xinyu Hong ${ }^{a, b}, c, d \quad$ Lingning Huang ${ }^{a, b}, c, d \quad$ Yongze Zhang a, b, c, d Ximei Shen ${ }^{a, b}, c, d$ \\ Suiyan Weng ${ }^{a, b, c, d}$ Feihui Zeng a, b, c, d Fengying Zhao a, b, c, d Sunjie Yan ${ }^{\text {a, b, c, d }}$ \\ ${ }^{a}$ Department of Endocrinology, The First Affiliated Hospital of Fujian Medical University, Fuzhou, China; ${ }^{\text {b Fujian }}$ \\ Province Clinical Research Center for Metabolic Diseases, Fuzhou, China; 'Diabetes Research Institute of Fujian \\ Province, Fuzhou, China; ${ }^{\mathrm{d} M e t a b o l i c}$ Diseases Research Institute, The First Affiliated Hospital of Fujian Medical \\ University, Fuzhou, China
}

\section{Keywords}

Type 2 diabetes - Albuminuria · Normoalbuminuria .

Estimated glomerular filtration rate $\cdot$ Vascular

complications

\begin{abstract}
Introduction: Albuminuria is a risk factor for macro- and microvascular complications of type 2 diabetes (T2D).With an increasing trend of normoalbuminuria, however, of the 2 predictors - estimated glomerular filtration rate (eGFR) and urine albumin-to-creatinine ratio (UACR) - which one is a better predictor of vascular complications of T2D is not clear. Objective: This study aimed to compare the impacts of albuminuria and eGFR on patients with T2D associated with micro- and macrovascular complications. Methods: This retrospective study recruited 4,715 patients with T2D and grouped them based on the values of UACR (high UACR: $\geq 30$ $\mathrm{mg} / \mathrm{g}$, low UACR: $<30 \mathrm{mg} / \mathrm{g})$ and eGFR $\left(\mathrm{mL} /\left[\mathrm{min} \times 1.73 \mathrm{~m}^{2}\right]\right)$ (G1: eGFR $\geq 90$; G2: eGFR = 60-89; G3-5: eGFR < 60) from April 2008 to November 2018. Logistic regression analysis was carried out for risk factors in patients with diabetic retinopathy (DR), diabetic peripheral neuropathy (DPN), peripheral arterial disease (PAD), left ventricular remodeling, diastolic disorders, and carotid atherosclerotic plaque in 6 different groups: low UACR + G1 (control group), low UACR +
\end{abstract}

G2, low UACR + G3-5, high UACR + G1, high UACR + G2, and high UACR + G3-5. Patients were grouped according to the change in the UACR value (UACR-decreased group: $\leq-30 \%$, UACR-stable group: -30 to $30 \%$, and UACR-increased group $\geq 30 \%$ ), eGFR value (eGFR-decreased group: >3\%, and eGFRstable group: $\leq 3 \%$ ) and followed up. Results: Compared with the control group, patients with higher albuminuria and lower eGFR had higher adjusted odds ratio (OR) trends of complications, especially in the high UACR + G3-5 group. The OR of 2.010, 3.444, 1.633, 2.742, and 3.014 were obtained for DR, DPN, PAD, left ventricular remodeling, and diastolic disorders, respectively. No statistically significant difference was found in the risk of complications within each one of 2 phenotypes, regardless of the change in the eGFR. After grouping by eGFR, the regression analysis of the urinary protein level in each stage revealed that a majority of complications had a statistically significant difference, except for DR and PAD in the high UACR + G3-5 group. DR in the follow-up study had a higher risk in the UACR-stable/increased group than the UACR-decreased group (UACR stable: OR = 2.568;

X.H., L.H, and Y.Z. share first authorship.

X.H. present address: Department of Endocrinology, The First Affiliated Hospital of Fujian Medical, now working at Fujian Medical University, Fuzhou, Fujian, China
(C) 2021 The Author(s)

Published by S. Karger AG, Basel

This is an Open Access article licensed under the Creative Commons Attribution-NonCommercial-4.0 International License (CC BY-NC) (http://www.karger.com/Services/OpenAccessLicense), applicable to the online version of the article only. Usage and distribution for commercial purposes requires written permission.
Correspondence to:

Sunjie Yan, fjyansunjie@163.com 
95\% confidence interval (Cl): 1.128-5.849; $p=0.025$; UACR increased: $\mathrm{OR}=2.489 ; 95 \% \mathrm{Cl}: 1.140-5.433 ; p=0.022)$. Con-

clusion: UACR is a more predictive risk factor for diabetic complications compared with a reduced eGFR.

(C) 2021 The Author(s).

Published by S. Karger AG, Basel

\section{Introduction}

Diabetes significantly increases the rates of many associated vascular complications, including cardiovascular diseases, retinopathy, neuropathy, nephropathy, and atherosclerosis, resulting in damage to corresponding target organs [1-3], decreased quality of life, and increased mortality [4]. No effective treatment is available once the diagnosis is made. The aforementioned longterm complications result in high mortality rates of patients with diabetes. Early intervention can prevent or at least delay disease progression in the subclinical stage. Thus, early biomarkers need to be urgently developed to halt disease progression.

Diabetic kidney disease (DKD), a long-term complication of diabetes, is the leading cause of end-stage kidney disease (ESKD) [5-7]. Previous studies have shown that both chronic kidney disease (CKD) with reduced renal function (based on estimated glomerular filtration rate [eGFR]) and albuminuria predict cardiovascular events in the general population and patients with type 2 diabetes (T2D) $[8,9]$. Albuminuria is an independent CV risk factor in nondiabetic individuals also, suggesting that it is not only a marker of early-stage DKD but also a major prognostic indicator in patients with diabetes. On the contrary, renal impairment, defined as an eGFR $<60 \mathrm{~mL} /\left(\min \times 1.73 \mathrm{~m}^{2}\right)$, may also occur in the absence of albuminuria in patients with diabetes [10]. Change in eGFR is also associated with vascular outcomes [11]. As a result, screening for kidney disease using the urinary albumin level and eGFR has become a cornerstone of diabetes care and targeted facilitation interventions to prevent cardiovascular disease and kidney disease progression. Albuminuria and eGFR may provide additional prognostic information regarding health outcomes in diabetes. eGFR decline is an endpoint for clinical trials in CKD. An increase of $30 \%$ in albuminuria is a subsequent risk factor for ESKD $[3,12]$. However, few studies investigated albuminuria and eGFR simultaneously. Hence, the joint relationship of albuminuria and eGFR with vascular diseases has not been elaborated, and different conclusions have been obtained [13, 14]. Wu et al. [15] investigated the relationship between $\mathrm{DR}$, neuropathy, and low ankle-brachial index (ABI) in patients with T2D ac- companied by mild to moderate $\mathrm{CKD}$. They found that the prevalence of diabetic retinopathy (DR) was graded according to the decline in the eGFR in patients with albuminuria, while the prevalence of peripheral arterial disease (PAD) gradually increased in patients with normoalbuminuria [15]. However, further research is required to confirm the results with large sample sizes and analyze the factors influencing complications of diabetes mellitus. Another study was conducted in Taiwan [16] and demonstrated that albuminuria was a stronger risk factor for PAD than a decline in eGFR in a Taiwanese population with T2D. However, no study focused on left ventricular remodeling and diastolic disorder. Biologic and analytic variabilities were greater for albuminuria than those for the eGFR, and the risk associations with the clinical endpoint were not as strong for albuminuria as for the eGFR decline. The present study combined the 2 different staging systems of DKD and found that macroalbuminuria was a stronger predictor of vascular complications than the reduction in the eGFR in patients with mild DKD.

\section{Methods}

\section{Study Subjects}

A retrospective study was conducted on 4,715 patients diagnosed with T2D, who were consecutively enrolled at the Department of Endocrinology and Metabolism of the First Affiliated Hospital of Fujian Medical University (Fuzhou, China) from April 2008 to November 2018. The exclusion criteria were as follows: (1) other types of diabetes (type 1 diabetes and gestational diabetes), (2) acute complications (diabetic ketoacidosis and hyperosmolar nonketotic comas), (3) other causes of renal disease (alcohol, nutrition, uremic, poisoning, acute infection, and drug addictions), (4) patients in critical condition or with end-stage cancer, and (5) patients with sodium-glucose cotransporter 2 inhibition. Patients were grouped based on the values of the urine albumin-to-creatinine ratio (UACR) and eGFR (high UACR: $\geq 30 \mathrm{mg} / \mathrm{g}$ or low UACR: $<30$ $\mathrm{mg} / \mathrm{g}$; G1: eGFR $\geq 90 \mathrm{~mL} /\left[\mathrm{min} \times 1.73 \mathrm{~m}^{2}\right], \mathrm{G} 2: \mathrm{eGFR}=60-89 \mathrm{~mL} /$ $\left[\mathrm{min} \times 1.73 \mathrm{~m}^{2}\right]$, and G3-5: eGFR $\left.<60 \mathrm{~mL} /\left[\mathrm{min} \times 1.73 \mathrm{~m}^{2}\right]\right)$ [17]. The patients with low UACR + G1 served as the control group.

\section{Medical Records}

The study subjects' medical records including height, weight, age, sex, smoking history, drinking history, diabetic duration, blood pressure (BP)-lowering medication (a total of 1,165 patients received renin-angiotensin-aldosterone system inhibitors), use of statin, and hypoglycemic agents were recorded. BP was measured twice where the study subjects were in sitting position after $15 \mathrm{~min}$ of rest, and the mean value of the 2 measurements was calculated

\section{Laboratory Investigations}

Blood samples were collected for measuring the levels of serum creatinine ( $\mathrm{sCr}$ ), uric acid (UA), albumin, triglyceride (TG), total cholesterol, high-density lipoprotein cholesterol (HDL-C), lowdensity lipoprotein cholesterol (LDL-C), very-low-density lipo- 
protein cholesterol, and fasting plasma glucose (FPG) collected overnight. Glycated hemoglobin A1c was examined using an automated processor (VARIANTTM II; BIO-RAD Laboratories Inc., Hercules, CA, USA). UACR was also calculated, and the CKD Epidemiology Collaboration (CKD-EPI) equation [18] was used to calculate the eGFR as follows:

$\mathrm{eGFR}=141 \times \min (\mathrm{sCr} / \kappa, 1)^{\alpha} \max (\mathrm{sCr} / \kappa, 1)^{-1.209} \times 0.993^{\text {age }}$ $[\times 1.018$ if female].

\section{Imaging Tests}

All participants underwent an ultrasound examination by an experienced ultrasonologist. Color Doppler ultrasound (ProSound a-10; Hitachi Aloka Medical, Ltd., Tokyo, Japan) was used to measure intima-media thickness [19], and echocardiography was additionally performed (GE-Vingmed Ultrasound, Horden, Norway); besides, left ventricular mass (LVM) was estimated based on the mean of 5 measurements of LV diameter and wall thickness. LVM was calculated using the American Society of Echocardiography-recommended linear formula involving left ventricular posterior wall thickness (LVPWT), interventricular septal wall thicknesses (IVST), and LV internal diameter at end diastole (LVDd):

$$
\operatorname{LVM}(\mathrm{g})=0.8 \times\left[1.04 \times(\mathrm{LVDd}+\mathrm{LVPWT}+\mathrm{IVST})^{3}-(\mathrm{LVDd})^{3}\right]
$$
+0.6 .

To remove the effects of volume, the LVM index (LVMI) was calculated as the LVM divided by the body surface area:

Relative wall thickness $(\mathrm{cm})=2 \times$ LVPWT/LVDd.

The ABI was measured by the systolic BP (SBP) from both brachial arteries and from both dorsalis pedis and posterior tibial arteries after the patient had been at rest in the supine position for $10 \mathrm{~min}$ [20].

\section{Complications}

Diabetic peripheral neuropathy (DPN) was defined as "symmetrical, length-dependent sensorimotor polyneuropathy attributable to chronic hyperglycemia, associated cardiovascular risk covariates, and microvessel alterations" [21]. When clinical features were atypical and the diagnosis was unclear, the disease was confirmed by referring to a neurologist.

DR was classified as a microvascular damage disease, with 2 subtypes: nonproliferative DR and proliferative DR [22]. An atherosclerotic plaque is demonstrated as a thickness of $>1.3 \mathrm{~mm}(0.13 \mathrm{~cm})$ measured from the media-adventitia interface to the intima-lumen interface [19]. The ABI represents the ratio of the ankle to brachial systolic pressure and is recommended to be calculated by dividing the higher systolic pressure of the dorsalis pedis and tibialis posterior vessels at the ankle with the higher systolic pressures measured in the brachial artery in both arms. The value of $\mathrm{ABI}<0.9$ or a postexercise $\mathrm{ABI}$ decrease of $>20 \%$ indicated a diagnosis of PAD or low $\mathrm{ABI}$ [23].

\section{Follow-Up Study}

All 6 groups (mean follow-up time: 22 months) were included in this retrospective follow-up study according to vascular complications (DR group, DPN group, PAD group, LV remodeling group, diastolic disorders group, and carotid atherosclerotic plaque group) to study the risk of diabetic vascular complications in patients. Each group had complete data without its group complications at the first visit: patients, who had fundus examinations but without DR in the first visit, regardless of whether it had DR in the following visit, were defined as the DR group; we can deduce the rest of the complications from this. According to the change in the UACR value, each group was divided into 3 subgroups: $\leq-30$, -30 to 30 , and $\geq 30 \%$. The regression analysis was conducted after adjusting for confounders. For eGFR analysis, the patients were divided into 2 groups according to the change in the eGFR value: $>3$ and $\leq 3 \%$. The progression in complications was observed. $\mathrm{Pa}-$ tients who had albuminuria were also enrolled in another followup study; the detected rate was calculated in the 2 groups of patients (grouped according to whether they use angiotensin-converting enzyme inhibitors (ACEIs)/angiotensin receptor blocks (ARBs) or not).

\section{Statistical Analysis}

All analyses were performed using SPSS 18.0 software (IBM, Armonk, NY, USA). Data were presented as mean \pm standard deviation, median (interquartile range), or proportions. All variables were tested for normal distribution using the Kolmogorov-Smirnov test. Patients were divided into 6 groups according to values of the UACR and eGFR. Statistically significant differences between the groups were determined using the $\chi^{2}$ test for categorical variables and analysis of variance for continuous variables, followed by the Bonferroni test. The binary regression model was used to study the relationship between the groups in terms of microvascular (DPN and DR) and macrovascular complications (carotid atherosclerotic plaque and $\mathrm{PAD}$ ). The odds ratio (OR) and 95\% confidence interval (CI) were calculated with reference to the control group. The levels of urinary protein and eGFR were stratified for retrospective analysis. A $p$ value $<0.05$ indicated statistically significant difference. In the followup study, the regression analysis was performed on patients in the following visits.

\section{Results}

\section{Patients' Clinical Characteristics}

The clinical data are shown in Table 1, according to the albuminuria level and CKD stage. For this purpose, 4,715 patients with T2D were recruited and grouped based on the values of the UACR and eGFR (high UACR $\geq 30 \mathrm{mg} / \mathrm{g}$ or low UACR $<30 \mathrm{mg} / \mathrm{g}$; G1: eGFR $\geq 90 \mathrm{~mL} /[\mathrm{min} \times 1.73$ $\left.\mathrm{m}^{2}\right], \mathrm{G} 2: \mathrm{eGFR}=60-89 \mathrm{~mL} /\left[\mathrm{min} \times 1.73 \mathrm{~m}^{2}\right], \mathrm{G} 3-5: \mathrm{eGFR}$ $\left.<60 \mathrm{~mL} /\left[\mathrm{min} \times 1.73 \mathrm{~m}^{2}\right]\right)$. Patients with a normal UACR at baseline had higher mean age, higher baseline mean eGFR, and lower serum UA level than patients with an increased UACR. With a reduction in the eGFR, the general trend indicated that the duration of diabetes, SBP, and the use of insulin and renin-angiotensin-aldosterone system inhibitors increased in both phenotypes (with or without albuminuria, as shown in online suppl. Fig. 1; see www.karger.com/doi/10.1159/000515163 for all online suppl. material). Patients with albuminuria had a significantly higher frequency of smoking, drinking, and drug use, and also higher diabetes duration, SBP, DBP, ABI value, and FPG, blood lipid, and glycosylated hemoglobin levels (all $p<0.05$, Table 1; online suppl. Fig. 1). 


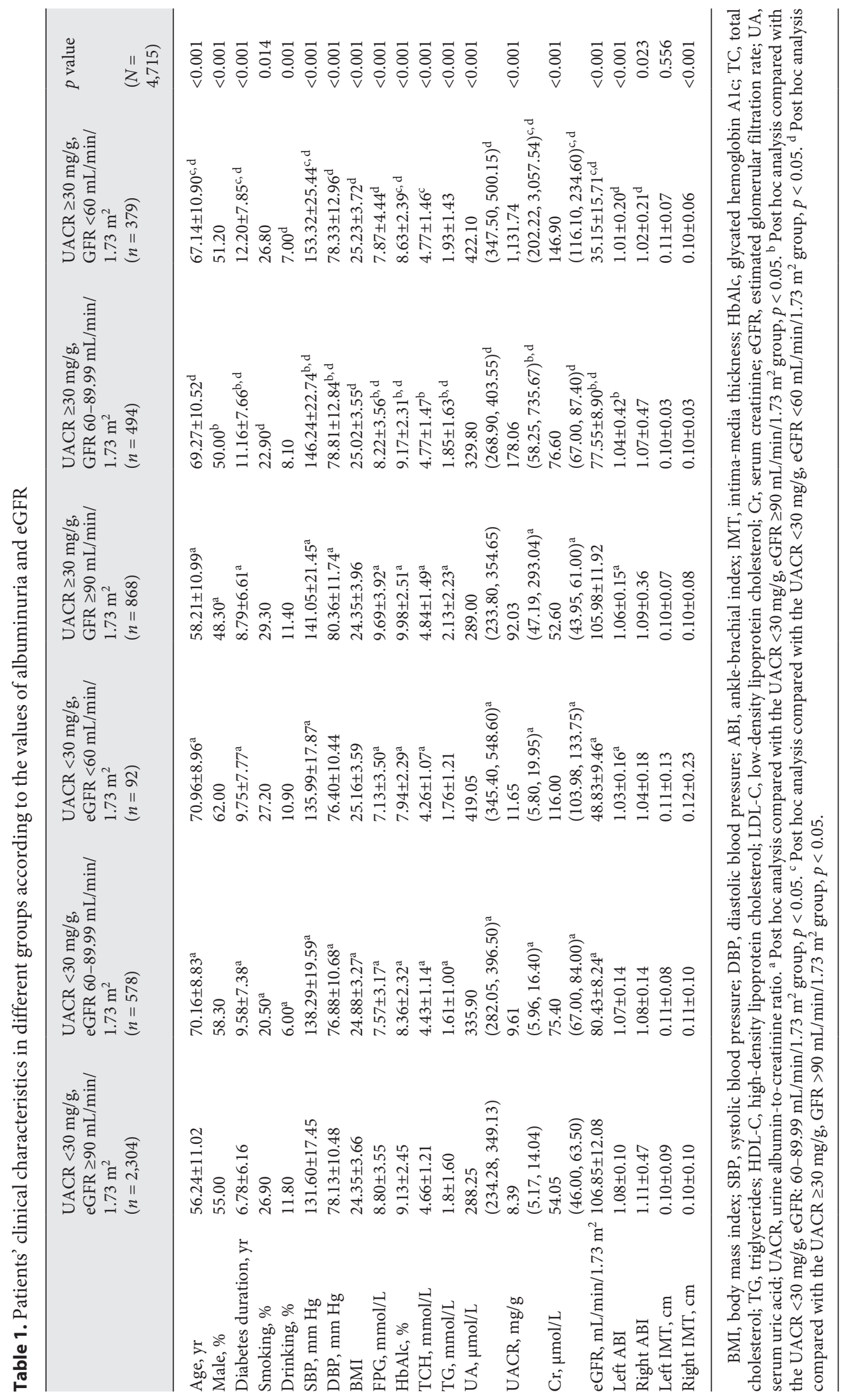


Detection of Diabetic Complications in Different

Stages with or without Albuminuria

Statistically significant differences were found in detecting DR, DPN, PAD, LV remodeling, and diastolic disorders between the albuminuria and normoalbuminuria groups from G1 to G3-5 in those with diabetes. The detection rates of DR, DPN, PAD, LV remodeling, and diastolic disorders were relatively lower (from G1 to G3-5) in patients with normoalbuminuria than in patients with albuminuria, as shown in Figure 1.

\section{Binary Regression Analysis for Vascular}

Complications in Patients with Diabetes Having

Different Levels of UACR and eGFR

The vascular complications in patients with diabetes having different levels of UACR and eGFR were analyzed by binary regression analysis after adjusting for age; sex; duration of diabetes; smoking status; drinking status; glycosylated hemoglobin level; body mass index; levels of low-density lipoprotein, UA, total cholesterol, very-lowdensity lipoprotein, TG, albumin, FPG, and HDL; SBP and diastolic BP; and use of beta-blockers, hypoglycemic drugs, insulin, and statins (online suppl. Fig. 2). Patients with albuminuria had adjusted ORs of 2.150 for G1 (95\% CI: $1.642-2.814$; $p<0.001), 2.356$ for G2 (95\% CI: 1.6493.367; $p<0.001)$, and 2.010 for G3-5 in DR (95\% CI: 1.318-3.067, $p=0.001$; online suppl. Fig. 2) compared with individuals in the control group, However, no significant differences were observed in patients with normoalbuminuria in different eGFR groups (OR: 1.323, 95\% CI: $0.933-1.876, p=0.116$; OR: 1.272 ; $95 \%$ CI: $0.602-$ $2.690, p=0.528$; online suppl. Fig. 2).

Similarly, the binary regression analysis was performed to assess the risk of DPN. Patients in the highest quintile with the urinary protein level had an adjusted OR of 3.444 (95\% CI: 2.019-5.875; online suppl. Fig. 2) for DPN compared with patients with the urinary protein level in the lowest quintile with an eGFR $\geq 90 \mathrm{~mL} /$ $\left(\min \times 1.73 \mathrm{~m}^{2}\right)$. A similar trend was observed in patients with PAD; the disease prevalence was higher in each stage in patients with albuminuria than in those with normoalbuminuria. For LV remodeling, the patients in the albuminuria group experienced an increase in ORs of LV remodeling (OR: 1.533, 95\% CI: $1.206-$ 1.949; OR: 1.945, 95\% CI: 1.413-2.676; OR: 2.742 , 95\% CI: 1.837-4.093; all $p<0.001$; online suppl. Fig. 2) compared with those in the control group. Similar results were obtained for diastolic disorders. The patients in the albuminuria group in different stages (from G1 to G3-5) had adjusted ORs of 1.777 (95\% CI: 1.223-2.581, $p=$
0.003 ), 2.054 (95\% CI: $1.317-3.204, p=0.002$ ), and 1.633 (95\% CI: $0.950-2.807, p=0.076$ ) for PAD compared with the individuals in the control group, as shown in online suppl. Figure 2. Additionally, the rates of DPN, $\mathrm{PAD}, \mathrm{LV}$ remodeling, diastolic disorders, and PAD were not statistically significantly different in the normoalbuminuria group, regardless of kidney function. The patients with a higher eGFR had a lower risk of PAD than individuals in the control group, but this trend was not observed for patients with an eGFR $<60 \mathrm{~mL} /(\mathrm{min} \times 1.73$ $\mathrm{m}^{2}$ ) in the albuminuria group. The results showed that the patients in the albuminuria group had a significantly higher risk with a lower GFR, while opposite results were obtained in patients without urinary protein (online suppl. Fig. 2).

\section{Regression Analysis of Levels of Urinary Protein and eGFR in Diabetics}

The vascular complications in patients with diabetes were compared according to the levels of proteinuria and eGFR. An increased risk of complications was observed with a decrease in the eGFR and improvement in the UACR level. However, whether the increased risk of complications was caused by urinary protein or decreased eGFR still remained elusive. Therefore, further regression analyses were conducted. When the patients were divided into 2 groups according to the presence of albuminuria, regression analysis showed no statistical difference in the risk of complications in each one of 2 phenotypes regardless of how eGFR changed (Fig. 2a, b). After grouping by eGFR, the regression analysis of the urinary protein in each stage revealed that the majority of complications had a statistical difference (Fig. 3). The pattern of association was similar to that of the baseline proteinuria because the complications were relatively serious in $\mathrm{DR}$ and diabetic peripheral vascular disease with a eGFR $<30 \mathrm{~mL} /(\mathrm{min} \times$ $\left.1.73 \mathrm{~m}^{2}\right)$.

\section{Follow-Up Study}

Regression Analysis on Vascular Complications

The regression analysis showed that the risk of DR significantly increased in patients with a UACR $\geq 30 \%$, with a higher risk in the UACR-stable/increased group than in the UACR-decreased group (UACR-stable: $\mathrm{OR}=2.568$, 95\% CI: $1.128-5.849, p=0.025$; UACR increased: OR $=$ 2.489 , 95\% CI: $1.140-5.433, p=0.022$ ) (online suppl. Fig. $3)$. The change in the eGFR was insignificant in the groups; other complications were stable, regardless of the change in the UACR or eGFR value. 


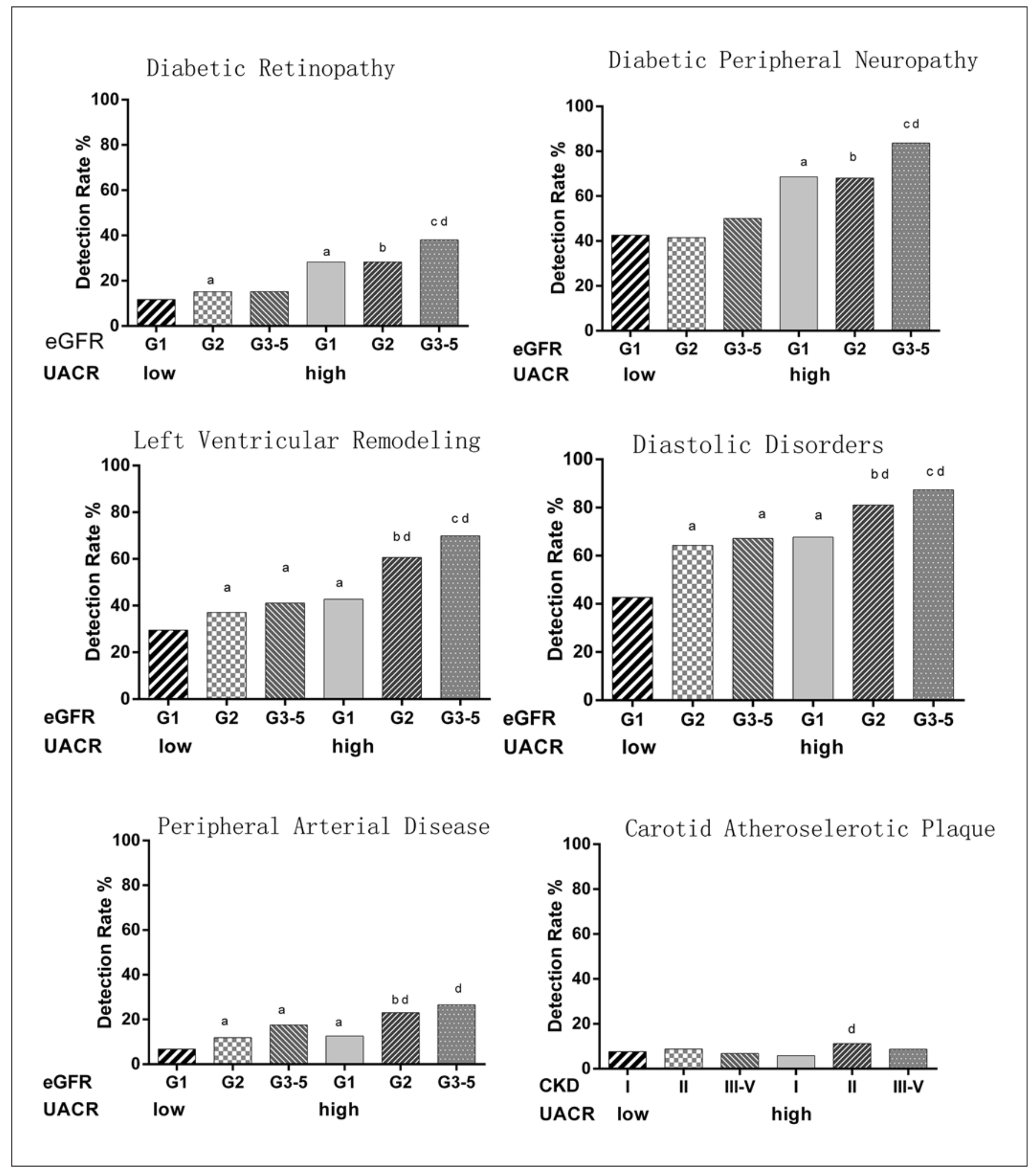

Fig. 1. Detection rate of vascular complications. Post hoc analysis: compared with the low UACR + G1, $p<0.05$ (a); compared with the low UACR + G2 (b); compared with the low UACR + G3-5, $p<0.05$ (c); compared with the high UACR + G1, $p<0.05$ (d).
UACR, urine albumin-to-creatinine ratio; eGFR, estimated glomerular filtration rate; $\mathrm{CKD}$, chronic kidney disease; LV, left ventricular; PAD, peripheral arterial disease. 
Normoalbuminuria (low UACR)

G1 vs G2

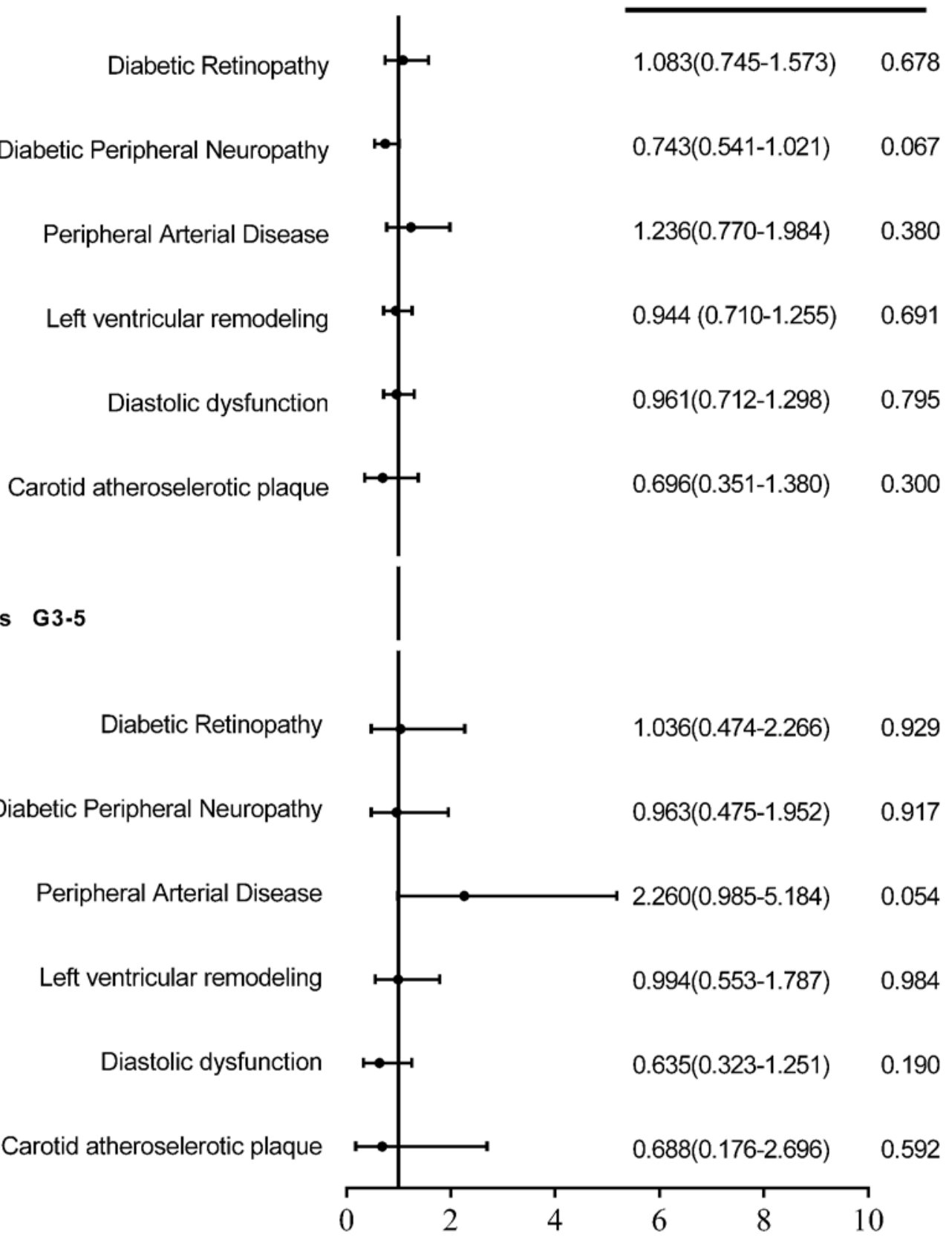

a

Forest plot of diabetic complications in normoalbuminuria

(Figure continued on next page.) 


\section{Albuminuria (high UACR)}

G1 vs G2

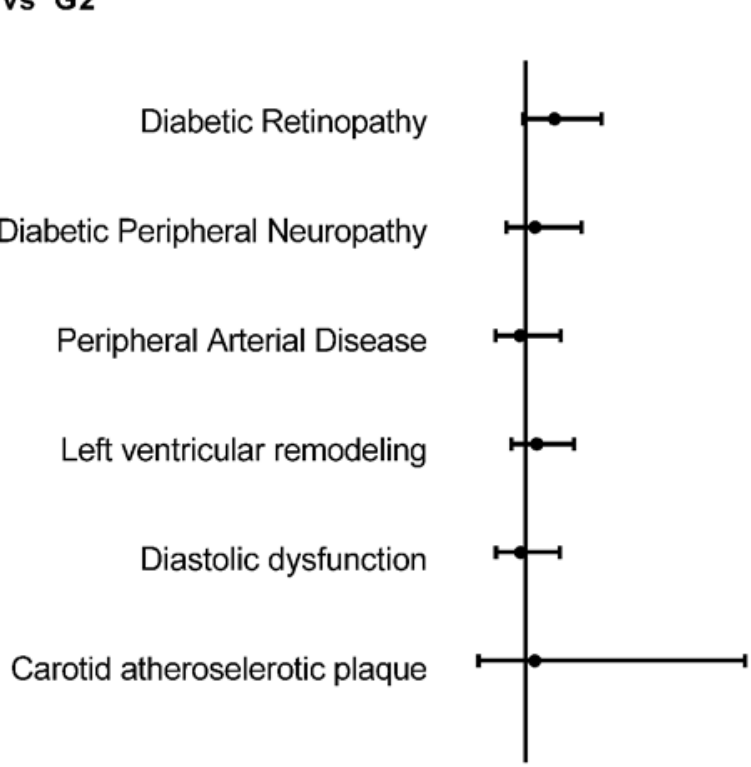

\begin{tabular}{cc} 
OR $(95 \% \mathrm{CI})$ & $\mathrm{P}$ \\
\hline $1.423(0.957-2.117)$ & 0.082 \\
& \\
$1.138(0.711-1.821)$ & 0.590 \\
$0.914(0.550-1.518)$ & 0.728 \\
& \\
$1.160(0.789-1.706)$ & 0.449 \\
$0.917(0.562-1.498)$ & 0.730 \\
& \\
$1.128(0.300-4.242)$ & 0.859
\end{tabular}

G1 vs $\mathbf{G} 3-5$

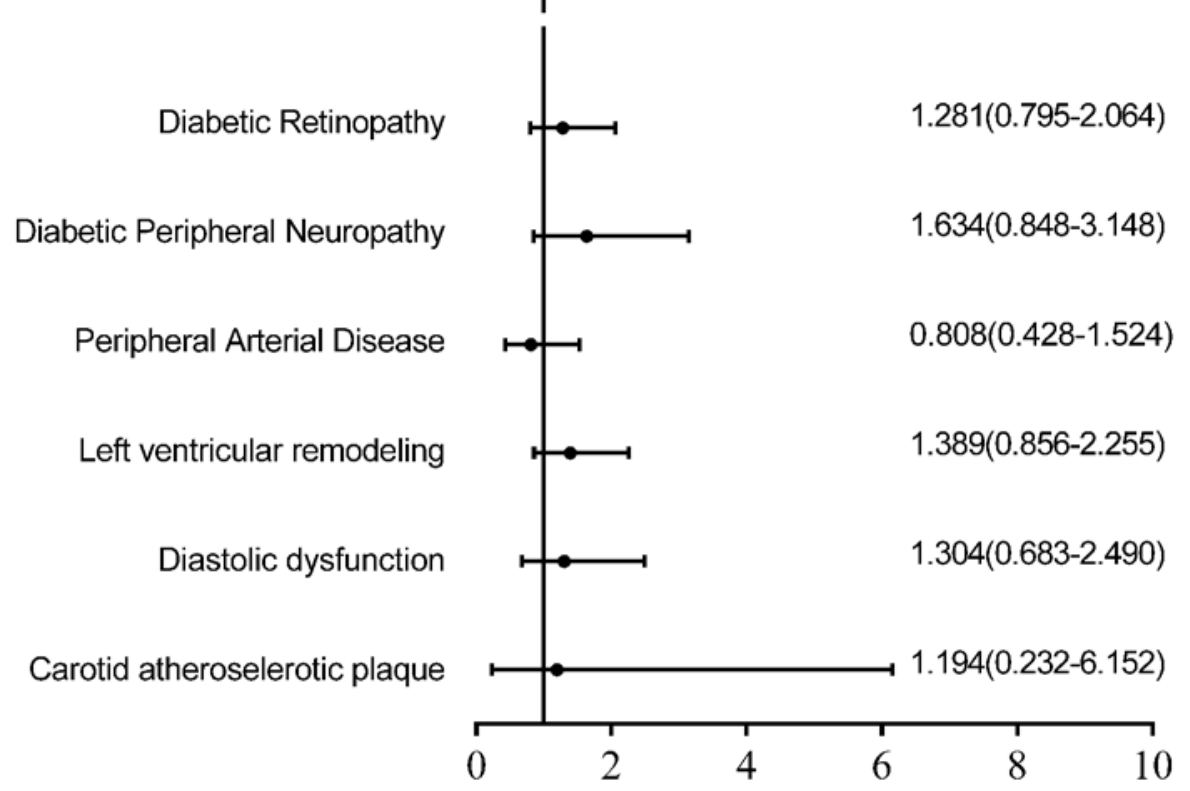

Forest plot of diabetic complications in albuminuria

Fig. 2. a Binary regression analysis of vascular complications in diabetics according to different stages of kidney function with urinary excretion of protein. $\mathbf{b}$ Binary regression analysis of vascular complications in diabetics according to different stages of kidney function without urinary excretion of protein. DR, diabetic retinopathy; DPN, diabetic peripheral neuropathy; UACR, urine albumin-to-creatinine ratio; $\mathrm{PAD}$, peripheral arterial disease; $\mathrm{OR}$, odds ratio; $\mathrm{CI}$, confidence interval; LV, left ventricular. 


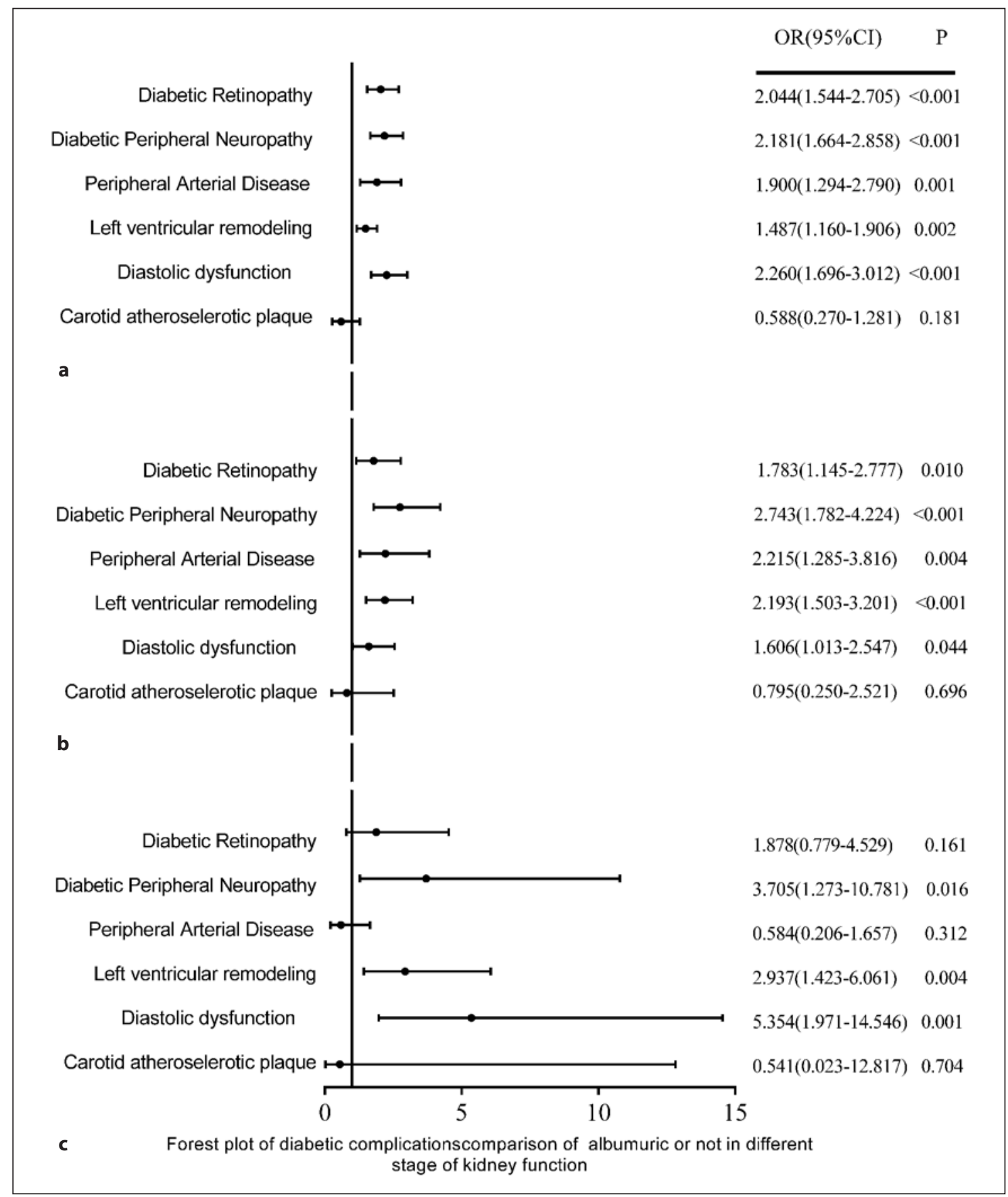

Fig. 3. Binary regression analysis of vascular complications in diabetics according to different stages of kidney function with different levels of urinary protein. Patients in group of G1 (a); patients in group of G2 (b); patients in group of G3-5 (c). DR, diabetic

Use of ACEIs or ARBs Had a Negative Correlation with the Detection Rate of Vascular Complications Patients who used ACEIs or ARBs during the follow-up period had a lower detection rate of vascular retinopathy; DPN, diabetic peripheral neuropathy; UACR, urine albumin-to-creatinine ratio; $\mathrm{PAD}$, peripheral arterial disease; $\mathrm{OR}$, odds ratio; CI, confidence interval; LV, left ventricular.

complications, but with no significant difference even after performing regression analysis, as shown in online suppl. Figure 4. 


\section{Discussion}

The present study demonstrated that the detection of diabetic complications was highly important in patients with different values of albuminuria but the same range of glomerular filtration that declined even after adjusting for multiple confounders. This finding was different from the traditional concept of both albuminuria and eGFR having effects on micro- and macrovascular complications. Therefore, determining the risk factors of renal impairment and albuminuria is of great clinical significance.

\section{Features of Normoalbuminuria with Vascular \\ Complications}

Few reports described the clinical characteristics of normoalbuminuric DKD with vascular complications. A previous report compared these characteristics with the characteristics of patients with micro-/macroalbuminuria [15]. Tingjun Wang et al. [24] also reported that low-grade albuminuria is related to LV hypertrophy and diastolic dysfunction in patients with hypertension; we also find similar relation, but it did not show the joint relationship between albuminuria and eGFR. The present study adjusted for confounders and extended the findings by presenting a lower prevalence of vascular complications. The prevailing trend of normoalbuminuric DKD was partially due to drug use. A study found that remission to normoalbuminuria was associated with a reduced serum creatinine level during 7.8 years of follow-up in patients with T2D and microalbuminuria. Both ACEIsand ARBs could decrease the UACR, especially for RAS blocker, reducing the risk of ESKD, renoprotection may seem to be related to inhibition of podocyte apoptosis [25]. In this study, we also found the same trend. Besides, lower vascular complications and even progress in eGFR were detected when ACEIs or ARBs were used patients, which may confirm the importance of albuminuric reduction (shown in online suppl. Table 1). Antihypertensive therapy and improved glycemic control were independent predictors for the remission [26], which decreased albuminuria with the use of ACEIs or ARBs.

\section{Albuminuria Is a Stronger Risk Factor}

The risk of complications of diabetes mellitus had no close association with the development of eGFR in either the albuminuria subgroup or the normoalbuminuria subgroup, even after adjusting for confounders. Another cross-sectional study conducted in Japan showed that the frequencies of simple retinopathy and neuropathy did

Stronger Association of Albuminuria with Risk of Diabetic Complications than eGFR not vary in different renal impairment groups, which was in general agreement with the findings of the present study. On the contrary, statistically significant differences were found in the hazard ratios of different eGFR groups in the albuminuria subgroup in the present study, confirming the pivotal role of albuminuria in complications compared with the eGFR. Similarly, Koye et al. [27] reported that the absence of albuminuria was common in patients with diabetes having reduced eGFRs and associated with a much lower risk of ESKD, CKD progression, or rapid decline in eGFR compared with that in patients with albuminuria. The present study confirmed that UACR was more meaningful in predicting microvascular complications compared with low eGFR. It might be explained via different mechanisms. Albuminuria was proved to cause widespread vascular damage [28] and endothelial dysfunction with increased levels of von Willebrand factor and thrombomodulin [29], indicating that the UACR was vital in microvascular complications and partly in macrovascular complications. The decline in GFR reflected nephron loss and substantive fibrosis partly due to abnormalities in terms of high TG and low HDL levels, and atherosclerosis [30], indicating that chronic complications were closely associated with albuminuria from a mechanistic perspective and with eGFR through renal damage.

\section{Association between Low eGFR and Vascular Complications}

Although albuminuria is a stronger risk factor than the eGFR, it does not mean that the eGFR is irrelevant to diabetic vascular complications. Chinese patients with T2D having a reduced eGFR showed that patients had a high risk of developing cardiovascular endpoints and all-cause mortality, independent of albuminuria and metabolic control [31]. A number of studies demonstrated that both normoalbuminuria and albuminuria with a low eGFR were independently associated with PAD in elderly patients with T2D [14]. This finding suggested that the eGFR might not be germane to microangiopathy and be vital as the underlying pathology in macroangiopathy $[32,33]$, indicating that the eGFR might influence renal function through age-related and vascular events [30], which was consistent with the findings of a previous study on patients with diabetes having an eGFR $<60 \mathrm{~mL} /(\mathrm{min}$ $\times 1.73 \mathrm{~m}^{2}$ ) [34].

\section{Diabetic Complications of End-Stage DKD}

With regard to the risk factors for albuminuria, the relationship between DR and proteinuria has been con- 
firmed by a number of previous studies [35-37]. The present study confirmed that albuminuria might be considered a risk factor for retinopathy. Besides, the concordance of CKD with advanced DR was low in patients with T2D and end-stage DKD. DR with G3-5 group of renal function $\left(\mathrm{eGFR}<60 \mathrm{~mL} /\left[\mathrm{min} \times 1.73 \mathrm{~m}^{2}\right]\right)$ was associated with an insignificantly different risk of albuminuria compared with normoalbuminuria. This might be due to the lowest tertile stage of renal function; also, the eGFR was lower than $60 \mathrm{~mL} /\left(\min \times 1.73 \mathrm{~m}^{2}\right)$, reducing the difference [38]. PAD might also partly contribute to the interference in measurements. Regarding another microvascular complication of peripheral neuropathy, albuminuria with the G3-5 group of renal function had a higher significance value than normoalbuminuria, which might be related to the accuracy of the electromyogram. The follow-up visit also confirmed the significance of albuminuria in the DR group, while similar results could not be achieved for other complications probably due to the short duration of follow-up. The present study showed that the incidence of vascular complications increased, except for carotid plaque, with the increase in the urinary protein level and decrease in the GFR.

A limited number of studies demonstrated the risk factors for renal dysfunction in T2D in the presence or absence of albuminuria, intrinsic relation between eGFR and UACR, especially with respect to cardiac diastolic function and LV remodeling. The main limitations of the present study were that it lacked the main outcomes regarding the association of the incidence of ESKD or CVD with the eGFR. The second possible limitation was that it was conservative to choose the UACR as evaluation of albuminuria, based on the recent KDIGO guidelines, which diagnosed DKD using the UACR. It cannot be denied that eAER is more accurate than ACR in the previous study. The third one was that system review reported that ARBs may be preferred for diabetic patients with albuminuria for reducing risks of ESKD and the sCr level [39], but we classified ACEIs and ARBs as one category [40].

\section{Conclusion}

The present study indicated the prevalence of macroand microvascular complications in patients with T2D with or without proteinuria in different stages of chronic nephropathy. It also showed that proteinuria was a more predictive risk factor for diabetes-related complications than a reduced eGFR. Patients with T2D should routinely receive statins and antihypertensive therapy to reduce the incidence of cardio(vascular) diseases. Further, attention should be paid to albuminuria for screening vascular complications in the clinical practice.

\section{Acknowledgments}

We are grateful to the patients for their help and willingness to participate in the study.

\section{Statement of Ethics}

All participants signed the written informed consent forms prior to the start of the study. This study was approved by the Ethics Committee of the First Affiliated Hospital of Fujian Medical University, and written informed consent was obtained from the patients: MRCTA and ECFAH of FMU[2017]131. All procedures performed in studies involving human participants were in accordance with the ethical standards of the institutional and/or national research committee and with the 1964 Helsinki Declaration and its later amendments or comparable ethical standards.

\section{Conflict of Interest Statement}

All authors have no conflicts of interest to declare.

\section{Funding Sources}

The study was funded by the Central Government Special Funds for Local Science and Technology Development (2018L 3007) and the Diabetes Fund from Chinese Society of Microcirculation (TW-2018P002).

\section{Author Contributions}

X.H. and S.Y.: conceived the study concept. X.H., F.Z., S.W., and F.Z.: collected the record. X.H. and Y.Z.: analyzed the data. X.H.: drafted the report. S.Y., L.H., X.S., and Y.Z.: provided critical revisions for important intellectual content. All authors took part in the interpretation of the data. All authors have read and approved the final manuscript.

\section{Availability of Data Statement}

The data set supporting the results of this study is included within the article. 


\section{References}

1 Shah MS, Brownlee M. Molecular and cellular mechanisms of cardiovascular disorders in diabetes. Circ Res. 2016;118(11): 1808-29.

2 Nathan DM. Diabetes: advances in diagnosis and treatment. JAMA. 2015;314(10): 1052-62.

3 Coresh J, Heerspink HJL, Sang Y, Matsushita K, Arnlov J, Astor BC, et al. Change in albuminuria and subsequent risk of endstage kidney disease: an individual participant-level consortium meta-analysis of observational studies. Lancet Diabetes Endocrinol. 2019;7(2):115-27.

4 Santaguida PL, Balion C, Hunt D, Morrison $\mathrm{K}$, Gerstein $\mathrm{H}$, Raina $\mathrm{P}$, et al. Diagnosis, prognosis, and treatment of impaired glucose tolerance and impaired fasting glucose. Evid Rep Technol Assess. 2005;(128): $1-11$

5 Packham DK, Alves TP, Dwyer JP, Atkins $\mathrm{R}$, de Zeeuw D, Cooper M, et al. Relative incidence of ESRD versus cardiovascular mortality in proteinuric type 2 diabetes and nephropathy: results from the DIAMETRIC (Diabetes mellitus treatment for renal insufficiency consortium) database. Am J Kidney Dis. 2012;59(1):75-83.

6 Ritz E, Rychlík I, Locatelli F, Halimi S. Endstage renal failure in type 2 diabetes: a medical catastrophe of worldwide dimensions. Am J Kidney Dis. 1999;34(5):795-808.

7 Afkarian M, Zelnick LR, Hall YN, Heagerty PJ, Tuttle K, Weiss NS, et al. Clinical manifestations of kidney disease among US adults with diabetes, 1988-2014. JAMA. 2016;316(6):602-10.

8 Gerstein HC, Mann JF, Yi Q, Zinman B, Dinneen SF, Hoogwerf B, et al. Albuminuria and risk of cardiovascular events, death, and heart failure in diabetic and nondiabetic individuals. JAMA. 2001; 286(4):421-6.

9 Kramer HJ, Nguyen QD, Curhan G, Hsu CY. Renal insufficiency in the absence of albuminuria and retinopathy among adults with type 2 diabetes mellitus. JAMA. 2003; 289(24):3273-7.

10 Tsalamandris C, Allen TJ, Gilbert RE, Sinha A, Panagiotopoulos S, Cooper ME, et al. Progressive decline in renal function in diabetic patients with and without albuminuria. Diabetes. 1994;43(5):649-55.

11 Adler AI, Stevens RJ, Manley SE, Bilous RW, Cull CA, Holman RR. Development and progression of nephropathy in type 2 diabetes: the United Kingdom Prospective Diabetes Study (UKPDS 64). Kidney Int. 2003;63(1):225-32.
12 Levey AS, Inker LA, Matsushita K, Greene $\mathrm{T}$, Willis K, Lewis E, et al. GFR decline as an end point for clinical trials in CKD: a scientific workshop sponsored by the $\mathrm{Na}$ tional kidney foundation and the US Food and drug administration. Am J Kidney Dis. 2014;64(6):821-35.

13 Bruno G, Merletti F, Bargero G, Novelli G, Melis D, Soddu A, et al. Estimated glomerular filtration rate, albuminuria and mortality in type 2 diabetes: the Casale Monferrato study. Diabetologia. 2007;50(5):941-8.

14 Ninomiya T, Perkovic V, de Galan BE, Zoungas S, Pillai A, Jardine M, et al. Albuminuria and kidney function independently predict cardiovascular and renal outcomes in diabetes. J Am Soc Nephrol. 2009; 20(8):1813-21.

15 Wu D, Xuan Y, Ruan Y, Feng X, Zhu Y, Jia $C$, et al. Prevalence of macro- and microvascular complications in patients with type 2 diabetes and kidney disease with or without albuminuria in a single Chinese diabetes centre. Diab Vasc Dis Res. 2016; 13(1):21-30

16 Lee MY, Lin KD, Chang YH, Hsiao PJ, Shin SJ. Albuminuria is the stronger risk factor for peripheral arterial disease than eGFR decline in a type 2 diabetic Taiwanese population. Kidney Blood Press Res. 2010; 33(5):352-9.

17 Levin A, Stevens PE, Bilous RW, Coresh J, De Francisco ALM, De Jong PE, et al. Kidney disease: improving global outcomes (KDIGO) CKD work group. KDIGO 2012 clinical practice guideline for the evaluation and management of chronic kidney disease. Kidney Int Suppl. 2013;3(1):1-150.

18 Levey AS, Stevens LA, Schmid CH, Zhang YL, Castro AF 3rd, Feldman HI, et al. A new equation to estimate glomerular filtration rate. Ann Intern Med. 2009;150(9): 604-12.

19 Cuspidi C, Ambrosioni E, Mancia G, Pessina AC, Trimarco B, Zanchetti A. Role of echocardiography and carotid ultrasonography in stratifying risk in patients with essential hypertension: the Assessment of Prognostic Risk Observational Survey. J Hypertens. 2002;20(7):1307-14.

20 Zhang Y, Guo Y, Shen X, Zhao F, Yan S. Lower body mass index is not of more benefit for diabetic complications. J Diabetes Investig. 2019;10(5):1307-17.

21 Pop-Busui R, Boulton AJ, Feldman EL, Bril V, Freeman R, Malik RA, et al. Diabetic neuropathy: a position statement by the American diabetes association. Diabetes care. 2017;40(1):136-54.

22 Wilkinson CP, Ferris FL 3rd, Klein RE, Lee PP, Agardh CD, Davis M, et al. Proposed international clinical diabetic retinopathy and diabetic macular edema disease severity scales. Ophthalmology. 2003;110(9): 1677-82.
23 Aboyans V, Criqui MH, Abraham P, Allison MA, Creager MA, Diehm C, et al. Measurement and interpretation of the anklebrachial index: a scientific statement from the American heart association. Circulation. 2012;126(24):2890-909.

24 Wang T, Zhong H, Lian G, Cai X, Gong J, Ye C, et al. Low-grade albuminuria is associated with left ventricular hypertrophy and diastolic dysfunction in patients with hypertension. Kidney Blood Press Res. 2019;44(4):590-603.

25 Wang $\mathrm{K}, \mathrm{Hu}$ J, Luo T, Wang Y, Yang S, Qing $\mathrm{H}$, et al. Effects of angiotensin-converting enzyme inhibitors and angiotensin II receptor blockers on all-cause mortality and renal outcomes in patients with diabetes and albuminuria: a systematic review and meta-analysis. Kidney Blood Press Res. 2018;43(3):768-79.

26 Gaede P, Tarnow L, Vedel P, Parving HH, Pedersen O. Remission to normoalbuminuria during multifactorial treatment preserves kidney function in patients with type 2 diabetes and microalbuminuria. Nephrol Dial Transplant. 2004;19(11):2784-8.

27 Koye DN, Magliano DJ, Reid CM, Jepson C, Feldman HI, Herman WH, et al. Risk of progression of nonalbuminuric $\mathrm{CKD}$ to end-stage kidney disease in people with diabetes: the CRIC (Chronic renal insufficiency cohort) Study. Am J Kidney Dis. 2018;72(5):653-61.

28 Deckert T, Feldt-Rasmussen B, BorchJohnsen K, Jensen T, Kofoed-Enevoldsen A. Albuminuria reflects widespread vascular damage. The Steno hypothesis. Diabetologia. 1989;32(4):219-26.

29 Hirano T, Ookubo K, Kashiwazaki K, Tajima H, Yoshino G, Adachi M. Vascular endothelial markers, von Willebrand factor and thrombomodulin index, are specifically elevated in type 2 diabetic patients with nephropathy: comparison of primary renal disease. Clin Chim Acta. 2000;299(1-2): 65-75.

30 Yokoyama H, Sone H, Oishi M, Kawai K, Fukumoto Y, Kobayashi M. Prevalence of albuminuria and renal insufficiency and associated clinical factors in type 2 diabetes: the Japan Diabetes Clinical Data Management study (JDDM15). Nephrol Dial Transplant. 2009;24(4):1212-9.

31 So WY, Kong AP, Ma RC, Ozaki R, Szeto CC, Chan NN, et al. Glomerular filtration rate, cardiorenal end points, and all-cause mortality in type 2 diabetic patients. Diabetes care. 2006;29(9):2046-52.

32 Pugliese G. Updating the natural history of diabetic nephropathy. Acta Diabetol. 2014; 51(6):905-15.
Stronger Association of Albuminuria with Risk of Diabetic Complications than eGFR
Kidney Blood Press Res 2021;46:550-562 DOI: $10.1159 / 000515163$ 
33 Porrini E, Ruggenenti P, Mogensen CE, Barlovic DP, Praga M, Cruzado JM, et al. Non-proteinuric pathways in loss of renal function in patients with type 2 diabetes. Lancet Diabetes Endocrinol. 2015;3(5): 382-91.

34 Ekinci EI, Jerums G, Skene A, Crammer P, Power D, Cheong KY, et al. Renal structure in normoalbuminuric and albuminuric patients with type 2 diabetes and impaired renal function. Diabetes Care. 2013;36(11): 3620-6.

35 Murussi M, Baglio P, Gross JL, Silveiro SP. Risk factors for microalbuminuria and macroalbuminuria in type 2 diabetic pa- tients: a 9-year follow-up study. Diabetes Care. 2002;25(6):1101-3.

36 Risk factors for development of microalbuminuria in insulin dependent diabetic patients: a cohort study. Microalbuminuria Collaborative Study Group, United Kingdom. BMJ. 1993;306(6887):1235-9.

37 Retnakaran R, Cull CA, Thorne KI, Adler AI, Holman RR. Risk factors for renal dysfunction in type 2 diabetes: U.K. Prospective Diabetes Study 74. Diabetes. 2006;55(6):1832-9.

38 Penno G, Solini A, Zoppini G, Orsi E, Zerbini G, Trevisan R, et al. Rate and determinants of association between advanced retinopathy and chronic kidney disease in patients with type 2 diabetes: the renal insufficiency and cardiovascular events (RIACE) Italian multicenter study. Diabetes Care. 2012;35(11):2317-23.

39 Hong DS, Oh IH, Park JS, Lee CH, Kang $\mathrm{CM}$, Kim GH. Evaluation of urinary indices for albuminuria and proteinuria in patients with chronic kidney disease. Kidney Blood Press Res. 2016;41(3):258-66.

40 Palmer SC, Mavridis D, Navarese E, Craig JC, Tonelli M, Salanti G, et al. Comparative efficacy and safety of blood pressure-lowering agents in adults with diabetes and kidney disease: a network meta-analysis. Lancet. 2015;385(9982):2047-56. 